\title{
Relaxation time for nonlinear response of a Brownian particle subject to a step external force: Analytical solutions for one-dimensional models
}

\author{
Yu. P. Kalmykov \\ Centre d'Etudes Fondamentales, Université de Perpignan, 52 Avenue de Villeneuve, 66860 Perpignan Cedex, France \\ and Institute of Radio Engineering and Electronics of the Russian Academy of Sciences, Vvedenskii Square 1, Fryazino, \\ Moscow Region, 141120, Russian Federation \\ J. L. Déjardin* \\ Centre d'Etudes Fondamentales, Université de Perpignan, 52 Avenue de Villeneuve, 66860 Perpignan Cedex, France \\ W. T. Coffey \\ Department of Electronic and Electrical Engineering, Trinity College, Dublin 2, Ireland
}

(Received 1 November 1996)

\begin{abstract}
The nonlinear response to a step external force of a system with relaxational dynamics governed by a one-dimensional Fokker-Planck equation is considered. An exact analytical expression for the step response nonlinear relaxation time is derived in terms of an integral (which can be evaluated numerically). Applications to nonlinear problems concerning the dynamic Kerr effect, dielectric relaxation of liquid dielectrics, and magnetic relaxation of systems of single domain ferromagnetic particles are given. The results are compared with solutions previously obtained. [S1063-651X(97)08503-6]
\end{abstract}

PACS number(s): 05.40.+j, 76.20.+q, 77.22.Gm, 78.20.Jq

\section{INTRODUCTION}

A system initially in an equilibrium (stationary) state and suddenly disturbed by an external stimulus (e.g., by applying a step external field) will evolve into another equilibrium (stationary) state. Presently a satisfactory theory is available for linear response only where the energy of the system arising from the external stimulus is much lower than the thermal energy [1,2]. Here we need only linear (in the external stimulus) deviations of the expectation value of the dynamical variable of interest in the stationary state in order to evaluate the generalized susceptibility and/or response functions in terms of the appropriate equilibrium (stationary) correlation function. Linear response theory is widely used for an interpretation of nonequilibrium phenomena such as dielectric and magnetic relaxation, conductivity problems, etc.

Here we wish to study relaxation following a steplike stimulus in systems described by one-dimensional FokkerPlanck equations for the distribution function $W(x, t)$ of a variable $x[2]$,

$$
\frac{\partial}{\partial t} W=L_{\mathrm{FP}} W
$$

We shall therefore first summarize the principal results of linear response theory ([2], Chap. 7) for systems where the dynamics obey a diffusion equation like Eq. (1.1). Thus let us consider the Fokker-Planck operator $L_{\mathrm{FP}}$ of a system subject to a small perturbing force $F(t)$. On account of this, $L_{\mathrm{FP}}$ may be represented as

\footnotetext{
*Author to whom correspondence should be addressed.
}

$$
\begin{aligned}
L_{\mathrm{FP}} & =\frac{\partial}{\partial x}\left[D^{(2)}(x) e^{-V(x)+B(x) F(t)} \frac{\partial}{\partial x} e^{V(x)-B(x) F(t)}\right] \\
& =L_{\mathrm{FP}}^{0}(x)+L_{\mathrm{ext}}(x) F(t),
\end{aligned}
$$

with

$$
\begin{gathered}
L_{\mathrm{FP}}^{0}(x)=\frac{\partial}{\partial x}\left[D^{(2)}(x) e^{-V(x)} \frac{\partial}{\partial x} e^{V(x)}\right], \quad L_{\mathrm{FP}}^{0}(x) W_{0}(x)=0, \\
L_{\mathrm{ext}}(x)=\frac{\partial}{\partial x}\left[D^{(2)}(x) B^{\prime}(x)\right], \quad B^{\prime}(x)=\frac{d}{d x} B,
\end{gathered}
$$

where $L_{\mathrm{FP}}^{0}(x)$ is the Fokker-Planck operator in the absence of the perturbation, $W_{0}$ is the equilibrium (stationary) distribution function, $V$ is called a generalized (effective) potential [2], $D^{(2)}(x)$ is the diffusion coefficient, and $B(x)$ denotes a dynamical quantity. The step-off and step-on relaxation functions (when, on the one hand, a small constant force $F_{1}$ is suddenly switched off and, on the other hand, switched on at time $t=0$, respectively, statistical equilibrium having been achieved prior to the imposition of the stimulus in both instances) for a dynamic variable $A(x)$ are then

$$
\begin{gathered}
\langle A\rangle^{\mathrm{off}}(t)-\langle A\rangle_{0}=F_{1} C_{A B}(t), \\
\langle A\rangle^{\mathrm{on}}(t)-\langle A\rangle_{0}=F_{1}\left[C_{A B}(0)-C_{A B}(t)\right] \quad(t>0),
\end{gathered}
$$

where the quantity 


$$
\begin{aligned}
C_{A B}(t) & =\langle A(x(0)) B(x(t))\rangle_{0}-\langle A\rangle_{0}\langle B\rangle_{0} \\
& =\int_{x_{1}}^{x_{2}}\left[A(x)-\langle A\rangle_{0}\right] e^{L_{\mathrm{FP}}^{0}}\left[B(x)-\langle B\rangle_{0}\right] W_{0}(x) d x
\end{aligned}
$$

is the equilibrium (stationary) correlation function, and the symbols \langle\rangle and \langle\rangle$_{0}$ designate the statistical averages over $W$ and $W_{0}$, respectively, with $x$ defined in the range $x_{1}<x<x_{2}$. Furthermore, the spectrum of $\langle A\rangle(t)$ (ac response) is

$$
\langle A\rangle_{\omega}=F_{\omega}\left[C_{A B}(0)-i \omega \int_{0}^{\infty} C_{A B}(t) e^{-i \omega t} d t\right],
$$

where $\langle A\rangle_{\omega}$ and $F_{\omega}$ are the Fourier components of $\langle A\rangle(t)$ and $F(t)$, respectively. We remark that Eqs. (1.3) and (1.5) are particular examples of Kubo's linear response theory [1]. Moreover, an exact integral formula exists for the correlation time $\tau_{A}$ [defined as the area under the curve of the normalized autocorrelation function $C_{A A}(t)$ as is apparent from Eq. (1.4) for $A=B$ ]. This is [see Eq. (S9.14) in Ref. [2]]

$$
\begin{aligned}
\tau_{A}= & \frac{1}{C_{A A}(0)} \int_{0}^{\infty} C_{A A}(t) d t \\
= & \frac{1}{C_{A A}(0)} \int_{x_{1}}^{x_{2}} \frac{1}{D^{(2)}(x) W_{0}(x)} \\
& \times\left[\int_{x_{1}}^{x_{2}}\left[A\left(x^{\prime}\right)-\langle A\rangle_{0}\right] W_{0}\left(x^{\prime}\right) d x^{\prime}\right]^{2} d x .
\end{aligned}
$$

In contrast, nonlinear response theory has been much less well developed by reason of its inherent mathematicalphysical complexity (see, e.g., [3,4]). The calculation of the nonlinear response even for systems described by a single coordinate is a difficult task as there is no longer any connection between the step-on and step-off responses and the ac response because the response now depends on the precise nature of the stimulus - as no unique response function valid for all stimuli unlike linear response exists. Such results as have been obtained have mainly emerged either by perturbation theory or by numerical simulations. However, a few exact analytical solutions of particular nonlinear step response problems exist (e.g., [5-7]). We shall now demonstrate that it is possible to derive an exact general equation in terms of an integral [similar to Eq. (1.6)] for the nonlinear step response relaxation time of a system governed by onedimensional Fokker-Planck equation (1.1) just as in linear response [2].

\section{ANALYTIC EQUATION FOR THE NONLINEAR RESPONSE RELAXATION TIME}

We consider the one-dimensional Brownian movement of a particle subject to a potential $V(x)$, and we assume that the relaxational dynamics of the particle obeys the FokkerPlanck equation (1.1). Let us suppose that at time $t=0$ the value of the generalized potential $V$ is suddenly changed from $V_{\text {I }}$ to $V_{\text {II }}$ (e.g., by applying a strong external field or by a change in some parameter characterizing the system). We are interested in the relaxation of the system starting from an equilibrium (stationary) state I with a distribution function $W_{\mathrm{I}}(x)$ which evolves under the action of the stimulus to another equilibrium (stationary) state II with the distribution function $W_{\text {II }}(x)$. Our goal is to evaluate the relaxation time $\tau_{A}$ of a typical dynamical variable $A$. This problem is intrinsically nonlinear, because we assume that changes in the magnitude of the potential are now significant. Thus the concept of relaxation functions and relaxation times must be used rather than correlation functions and correlation times.

We define the normalized relaxation function $f_{A}(t)$ of a dynamical variable $A$ by

$$
f_{A}(t)=\left\{\begin{array}{l}
\frac{\langle A\rangle(t)-\langle A\rangle_{\mathrm{II}}}{\langle A\rangle_{I}-\langle A\rangle_{\mathrm{II}}}, \quad t>0 \\
1, \quad t \leqslant 0
\end{array}\right.
$$

where $\langle A\rangle_{\mathrm{I}}$ and $\langle A\rangle_{\mathrm{II}}$ are equilibrium (stationary) averages defined as

$$
\langle A\rangle_{\mathrm{I}}=\int_{x_{1}}^{x_{2}} A(x) W_{\mathrm{I}}(x) d x, \quad\langle A\rangle_{\mathrm{II}}=\int_{x_{1}}^{x_{2}} A(x) W_{\mathrm{II}}(x) d x,
$$

and $\langle A\rangle(t)$ is the time-dependent average,

$$
\langle A\rangle(t)=\int_{x_{1}}^{x_{2}} A(x) W(x, t) d x .
$$

The relaxation time $\tau_{A}$ defined as the area under the curve of $f_{A}(t)$ at $t>0$ is then given by

$$
\tau_{A}=\int_{0}^{\infty} f_{A}(t) d t=\lim _{s \rightarrow 0} \int_{0}^{\infty} e^{-s t} f_{A}(t) d t=\widetilde{f}_{A}(0),
$$

where $\widetilde{f}_{A}(s)$ is the Laplace transform of $f_{A}(t)$. On interchanging the orders of integration over $x$ and $t$ in Eq. (2.4), we have

$$
\tau_{A}=\frac{1}{\langle A\rangle_{\mathrm{I}}-\langle A\rangle_{\mathrm{II}}} \int_{x_{1}}^{x_{2}}\left[A(x)-\langle A\rangle_{\mathrm{II}}\right] \widetilde{W}(x, 0) d x,
$$

where

$$
\widetilde{W}(x, 0)=\lim _{s \rightarrow 0} \tilde{W}(x, s)
$$

and

$$
\widetilde{W}(x, s)=\int_{0}^{\infty} W(x, t) e^{-s t} d t .
$$

The quantity $\widetilde{W}(x, 0)$ can be calculated analytically by quadratures as follows. On using the final value theorem of Laplace transformation, viz.

$$
\lim _{s \rightarrow 0} s \tilde{W}(x, s)=\lim _{t \rightarrow \infty} W(x, t)=W_{\text {II }}(x),
$$

and on taking into account Eq. (1.2), from Eq. (1.1) at $t>0$ we obtain 


$$
\begin{aligned}
W_{\mathrm{II}}(x)-W_{\mathrm{I}}(x) & \\
= & \frac{d}{d x}\left[D^{(2)}(x)\left(\frac{d}{d x} \widetilde{W}(x, 0)+\widetilde{W}(x, 0) \frac{d}{d x} V_{\mathrm{II}}(x)\right)\right] .
\end{aligned}
$$

The solution of Eq. (2.8) is

$$
\widetilde{W}(x, 0)=W_{\mathrm{II}}(x) \int_{x_{1}}^{x} \frac{\Phi(y) d y}{D^{(2)}(y) W_{\mathrm{II}}(y)},
$$

where

$$
\Phi(y)=\int_{x_{1}}^{y}\left[W_{\mathrm{II}}(z)-W_{\mathrm{I}}(z)\right] d z .
$$

Thus from Eqs. (2.5) and (2.9) we obtain

$$
\begin{aligned}
\tau_{A}= & \frac{1}{\langle A\rangle_{\mathrm{I}}-\langle A\rangle_{\mathrm{II}}} \int_{x_{1}}^{x_{2}}\left[A(x)-\langle A\rangle_{\mathrm{II}}\right] W_{\mathrm{II}}(x) \\
& \times \int_{x_{1}}^{x} \frac{\Phi(y)}{D^{(2)}(y) W_{\mathrm{II}}(y)} d y d x
\end{aligned}
$$

so that, on integration by parts,

$$
\tau_{A}=\frac{1}{\langle A\rangle_{\mathrm{II}}-\langle A\rangle_{\mathrm{I}}} \int_{x_{1}}^{x_{2}} \frac{\Phi(x) \Psi(x)}{D^{(2)}(x) W_{\mathrm{II}}(x)} d x,
$$

where

$$
\Psi(x)=\int_{x_{1}}^{x}\left[A(y)-\langle A\rangle_{\mathrm{II}}\right] W_{\mathrm{II}}(y) d y .
$$

Equation (2.11) is an exact equation for the nonlinear step response relaxation time, which is analogous to Eq. (1.6) for the linear response.

\section{EXAMPLES}

As a first example, we consider the one-dimensional noninertial translational Brownian motion of a particle in a potential $V(x)$, where the variable $x$ specifies the position of the particle. The relevant Fokker-Planck (Smoluchowski) equation for the distribution function $W$ of the position $x$ is given by [2]

$$
\zeta \frac{\partial}{\partial t} W=\frac{\partial}{\partial x}\left(W \frac{\partial}{\partial x} V_{\mathrm{II}}(x)\right)+\frac{1}{\beta} \frac{\partial^{2}}{\partial x^{2}} W \quad(t>0),
$$

with the initial conditions $W(x, 0)=W_{\mathrm{I}}(x)$, where $\zeta$ is the friction coefficient and $\beta=1 / k T$. For potentials, where the probability current $S$ in the stationary state is not equal to zero [e.g., for the tilted periodic potential $\left.V(x)=V_{0}(\cos x-a x)\right]$, the relaxation time is given by Eq. (2.11), with the stationary distribution functions $W_{\mathrm{I}}(x)$ and $W_{\text {II }}(x)$ defined as [2]

$$
W_{i}(x)=N_{i} e^{-\beta V_{i}(x)}-S_{i} e^{-\beta V_{i}(x)} \int_{x_{0}}^{x} \frac{e^{\beta V_{i}(y)}}{D^{(2)}(y)} d y \quad(i=\mathrm{I}, \mathrm{II}),
$$

where

$$
D^{(2)}(x)=1 / \zeta \beta
$$

and the constants $N_{i}$ and $S_{i}$ must be determined from the boundary and the normalization conditions [2].

On the other hand, for potentials, where the probability current $S=0$ at the stationary state [e.g., for the bistable potential $\left.V(x)=-V_{0}\left(b x^{2}-x^{4}\right)\right], W_{\mathrm{I}}(x)$ and $W_{\mathrm{II}}(x)$ are the Maxwell-Boltzmann distribution functions, viz.

$$
W_{i}(x)=e^{-\beta V_{i}(x)} / Z_{i} \quad(i=\mathrm{I}, \mathrm{II}) .
$$

Here $Z_{\mathrm{I}}$ and $Z_{\mathrm{II}}$ are the partition functions, and $x_{1}=-\infty$ and $x_{2}=\infty$. The relaxation time is therefore given by

$$
\tau_{A}=\frac{\beta \zeta}{\langle A\rangle_{\mathrm{II}}-\langle A\rangle_{\mathrm{I}}} \int_{-\infty}^{\infty} e^{\beta V_{\mathrm{II}}(x)} \Phi(x) \Psi(x) d x,
$$

where

$$
\begin{gathered}
\Phi(z)=\int_{-\infty}^{z}\left[Z_{\mathrm{II}}^{-1} e^{-\beta V_{\mathrm{II}}(y)}-Z_{\mathrm{I}}^{-1} e^{-\beta V_{\mathrm{I}}(y)}\right] d y, \\
\Psi(z)=\int_{-\infty}^{z}\left[A(x)-\langle A\rangle_{\mathrm{II}}\right] e^{-\beta V_{\mathrm{II}}(x)} d x .
\end{gathered}
$$

Similar results are obtained for the longitudinal relaxation arising from the noninertial rotational Brownian motion of a dipolar particle in an external uniaxial potential $V$. The relevant Fokker-Planck equation for the distribution function $W$ of the orientations of the particle is $[8,9]$

$$
\begin{aligned}
2 \tau_{D} \frac{\partial}{\partial t} W= & \frac{\beta}{\sin \vartheta} \frac{\partial}{\partial \vartheta}\left[\sin \vartheta W \frac{\partial}{\partial \vartheta} V_{\mathrm{II}}(\vartheta)\right] \\
& +\frac{1}{\sin \vartheta} \frac{\partial}{\partial \vartheta}\left[\sin \vartheta \frac{\partial}{\partial \vartheta} W\right] \quad(t>0),
\end{aligned}
$$

( $\vartheta$ is the polar angle which specifies the orientation of the particle) or introducing a variable $x=\cos \vartheta$

$$
\begin{gathered}
2 \tau_{D} \frac{\partial}{\partial t} W=\frac{\partial}{\partial x}\left(\left(1-x^{2}\right)\left(\frac{\partial}{\partial x} W+\beta W \frac{\partial}{\partial x} V_{\mathrm{II}}(x)\right)\right) \\
(t>0)
\end{gathered}
$$

where $\tau_{D}$ is a characteristic (Debye) relaxation time. In this case $W_{\mathrm{I}}(x)$ and $W_{\mathrm{II}}(x)$ are the Maxwell-Boltzmann distribution functions

$$
W_{\mathrm{I}}(x)=e^{-\beta V_{\mathrm{I}}(x)} / Z_{\mathrm{I}}, \quad W_{\mathrm{II}}(x)=e^{-\beta V_{\mathrm{II}}(x)} / Z_{\mathrm{II}},
$$

$x_{1}=-1, x_{2}=1$, and

$$
D^{(2)}(x)=\frac{1-x^{2}}{2 \tau_{D}} .
$$

Thus Eq. (2.11) yields

$$
\tau_{A}=\frac{2 \tau_{D}}{\langle A\rangle_{\mathrm{II}}-\langle A\rangle_{\mathrm{I}}} \int_{-1}^{1} \frac{e^{\beta V_{\mathrm{II}}(z)} \Phi(z) \Psi(z) d z}{1-z^{2}},
$$


where

$$
\begin{gathered}
\Phi(z)=\int_{-1}^{z}\left[Z_{\mathrm{II}}^{-1} e^{-\beta V_{\mathrm{II}}(y)}-Z_{\mathrm{I}}^{-1} e^{-\beta V_{\mathrm{I}}(y)}\right] d y, \\
\Psi(z)=\int_{-1}^{z}\left[A(x)-\langle A\rangle_{\mathrm{II}}\right] e^{-\beta V_{\mathrm{II}}(x)} d x .
\end{gathered}
$$

\section{APPLICATION TO NONLINEAR DYNAMIC KERR EFFECT, DIELECTRIC AND MAGNETIC RELAXATION}

Equation (3.8) can be used to calculate the relaxation time of the nonlinear dielectric and dynamic Kerr effect step responses of systems consisting of permanently polar and polarizable molecules. It may also be applied to the calculation of the nonlinear relaxation time of nematic liquid crystals as well as single domain ferromagnetic particles where the amplitude of a strong dc field $\mathbf{F}_{0}$ (electric or magnetic as appropriate) is suddenly changed at $t=0$. The longitudinal relaxation of all these systems (excluding interparticle interactions) is governed by the Fokker-Planck equation (3.5), with a uniaxial potential $V$ given by

$$
\beta V(\cos \vartheta)=-\sigma \cos ^{2} \vartheta-\xi \cos \vartheta
$$

or

$$
\beta V(z)=-\sigma\left(z^{2}+2 h z\right) \quad(z=\cos \vartheta)
$$

where

$$
\sigma=\beta K, \quad \xi=\beta \mu F_{0} \quad \text { and } \quad h=\xi / 2 \sigma
$$

are the appropriate dimensionless anisotropy and external field parameters and their ratio, respectively, $K$ is an anisotropy constant, and $\mu$ is the (electric or magnetic) dipole moment. The appropriate interpretation of the parameters $\tau_{D}, \xi$, and $\sigma$ in each case is given, e.g., in Refs. [8-16]. The potential Eq. (4.1) is symmetrical when $\xi=0$ and has a barrier at $\vartheta=\pi / 2$, where the potential has a maximum where the height relative to the minima at $\vartheta=0$, and $\vartheta=\pi$ is equal to $\sigma$. The potential becomes asymmetrical for $\xi \neq 0$ and the double well structure disappears at $h=h_{s}=1$.

The quantities of greatest interest in the nonlinear response of these systems are the relaxation times $\tau_{n}(n=1$ and 2 ) of the relaxation functions $f_{1}(t)$ and $f_{2}(t)$ of the first and second Legendre polynomials, viz.

$$
f_{1}(t)=\left\langle P_{1}(\cos \vartheta)\right\rangle(t)-\left\langle P_{1}(\cos \vartheta)\right\rangle_{\mathrm{II}}
$$

and

$$
f_{2}(t)=\left\langle P_{2}(\cos \vartheta)\right\rangle(t)-\left\langle P_{2}(\cos \vartheta)\right\rangle_{\mathrm{II}}
$$

Equation (4.3) governs the dielectric and magnetic relaxation, and Eq. (4.4) governs the dynamic Kerr effect. The distribution functions in the equilibrium states I and II are given by

$$
W_{i}(z)=e^{\sigma_{i}\left(2 h_{i} z+z^{2}\right)} / Z_{i} \quad(i=\mathrm{I}, \mathrm{II}),
$$

where

$$
\begin{aligned}
Z_{i}= & \frac{1}{2}\left(\frac{\pi}{\sigma_{i}}\right)^{1 / 2} e^{-\sigma_{i} h_{i}^{2}}\left\{\operatorname{erf} i\left[\left(1+h_{i}\right) \sqrt{\sigma_{i}}\right]\right. \\
& \left.+\operatorname{erf} i\left[\left(1-h_{i}\right) \sqrt{\sigma_{i}}\right]\right\}
\end{aligned}
$$

is the partition function, and

$$
\operatorname{erf} i(x)=\frac{2}{\sqrt{\pi}} \int_{0}^{x} e^{t^{2}} d t
$$

is the error function of imaginary argument. From Eqs. (2.11), (4.5), and (4.6), we have

$$
\begin{gathered}
\tau_{n}=\frac{2 \tau_{D}}{\left\langle P_{n}\right\rangle_{\mathrm{II}}-\left\langle P_{n}\right\rangle_{\mathrm{I}}} \int_{-1}^{1} \frac{\Phi(z) \Psi_{n}(z) e^{-\sigma_{\mathrm{II}} z^{2}-\zeta_{\mathrm{II}}} d z}{1-z^{2}} \\
n=1 \text { and } 2
\end{gathered}
$$

where

$$
\begin{aligned}
\Phi(z)= & \int_{-1}^{z}\left[W_{\mathrm{II}}\left(z^{\prime}\right)-W_{\mathrm{I}}\left(z^{\prime}\right)\right] d z^{\prime}=\frac{\pi^{1 / 2} e^{-\sigma_{\mathrm{II}} h_{\mathrm{II}}^{2}}}{2 \sigma_{\mathrm{II}}^{1 / 2} Z_{\mathrm{II}}} \\
& \times\left\{\operatorname{erf} i\left[\left(z+h_{\mathrm{II}}\right) \sqrt{\sigma_{\mathrm{II}}}\right]+\operatorname{erf} i\left[\left(1-h_{\mathrm{II}}\right) \sqrt{\sigma_{\mathrm{II}}}\right]\right\} \\
& -\frac{\pi^{1 / 2} e^{-\sigma_{\mathrm{I}} h_{\mathrm{I}}^{2}}}{2 \sigma_{\mathrm{I}}^{1 / 2} Z_{\mathrm{I}}}\left\{\operatorname{erf} i\left[\left(z+h_{\mathrm{I}}\right) \sqrt{\sigma_{\mathrm{I}}}\right]\right. \\
& \left.+\operatorname{erf} i\left[\left(1-h_{\mathrm{I}}\right) \sqrt{\sigma_{\mathrm{I}}}\right]\right\}
\end{aligned}
$$

$$
\begin{aligned}
\Psi_{1}(z)= & \int_{-1}^{z}\left[P_{1}\left(z^{\prime}\right)-\left\langle P_{1}\right\rangle_{\mathrm{II}}\right] e^{\sigma_{\mathrm{II}}\left(z^{\prime 2}+2 h_{\mathrm{II}} z^{\prime}\right)} d z^{\prime} \\
= & \frac{1}{2 \sigma_{\mathrm{II}}}\left[e^{\sigma_{\mathrm{II}}\left(z^{2}+2 h_{\mathrm{II}} z\right)}-e^{\sigma_{\mathrm{II}}\left(1-2 h_{\mathrm{II}}\right)}\right] \\
& -e^{\sigma_{\mathrm{II}}\left(1-h_{\mathrm{II}}^{2}\right)} \frac{\pi^{1 / 2} \sinh \left(2 \sigma_{\mathrm{II}} h_{\mathrm{II}}\right)}{2 \sigma_{\mathrm{II}}^{3 / 2} Z_{\mathrm{II}}}\left\{\operatorname{erf} i\left[\left(z+h_{\mathrm{II}}\right) \sqrt{\sigma_{\mathrm{II}}}\right]\right. \\
& \left.+\operatorname{erf} i\left[\left(1-h_{\mathrm{II}}\right) \sqrt{\sigma_{\mathrm{II}}}\right]\right\}, \\
\Psi_{2}(z)= & \int_{-1}^{z}\left[P_{2}\left(z^{\prime}\right)-\left\langle P_{2}\right\rangle_{\mathrm{II}}\right] e^{\sigma_{\mathrm{II}}\left(z^{\prime 2}+2 h_{\mathrm{II}} z^{\prime}\right)} d z^{\prime} \\
= & \frac{3}{4 \sigma_{\mathrm{II}}}\left\{e^{\sigma_{\mathrm{II}}\left(z^{2}+2 h_{\mathrm{II}} z\right)}\left(z-h_{\mathrm{II}}\right)+e^{\sigma_{\mathrm{II}}\left(1-2 h_{\mathrm{II}}\right)}\left(1+h_{\mathrm{II}}\right)\right. \\
& -e^{\sigma_{\mathrm{II}}\left(1-h_{\mathrm{II}}^{2}\right)} \frac{\pi^{1 / 2}\left[\cosh \left(2 \sigma_{\mathrm{II}} h_{\mathrm{II}}\right)-h_{\mathrm{II}} \sinh \left(2 \sigma_{\mathrm{II}} h_{\mathrm{II}}\right)\right]}{\sigma_{\mathrm{II}}^{1 / 2} Z_{\mathrm{II}}} \\
& \left.\times\left[\operatorname{erf} i\left[\left(z+h_{\mathrm{II}}\right) \sqrt{\sigma_{\mathrm{II}}}\right]+\operatorname{erf} i\left[\left(1-h_{\mathrm{II}}\right) \sqrt{\sigma_{\mathrm{II}}}\right]\right]\right\},
\end{aligned}
$$

$$
\left\langle P_{1}\right\rangle_{i}=\frac{e^{\sigma_{i}} \sinh \left(2 \sigma_{i} h_{i}\right)}{\sigma_{i} Z_{i}}-h_{i}
$$




$$
\begin{aligned}
\left\langle P_{2}\right\rangle_{i}= & \frac{3 e^{\sigma_{i}}\left[\cosh \left(2 \sigma_{i} h_{i}\right)-h_{i} \sinh \left(2 \sigma_{i} h_{i}\right)\right]}{2 \sigma_{i} Z_{i}} \\
& +\frac{3 h_{i}^{2}}{2}-\frac{3}{4 \sigma_{i}}-\frac{1}{2} \quad(i=\mathrm{I}, \mathrm{II}) .
\end{aligned}
$$

First, we shall compare the foregoing results with those previously given. We have obtained in Refs. [6,7] by solving the infinite hierarchy of differential-recurrence relations for the averaged spherical harmonics, the exact analytical solution for the relaxation time $\tau_{2}$ for the nonlinear rise transient of the dynamic Kerr effect for nonpolar polarizable molecules, which is (in the notation of Ref. [7])

$$
\begin{aligned}
\tau_{2}= & \frac{45 \pi \tau_{D}}{64 M\left(\frac{1}{2}, \frac{3}{2}, \sigma\right) M\left(\frac{3}{2}, \frac{7}{2}, \sigma\right)} \sum_{n=1}^{\infty}\left(-\frac{\sigma}{2}\right)^{2 n-2} \\
& \times \frac{(4 n+1) \Gamma(2 n) M^{2}\left(n+\frac{1}{2}, 2 n+\frac{3}{2}, \sigma\right)}{(2 n+1) \Gamma^{2}\left(2 n+\frac{3}{2}\right)} .
\end{aligned}
$$

Here $\Gamma(z)$ is the gamma function [17], and $M(a, b, z)$ is the confluent hypergeometric (Kummer) function defined as [17]

$$
\begin{aligned}
M(a, b, z)= & +\frac{a}{b} \frac{z}{1 !}+\frac{a(a+1)}{b(b+1)} \frac{z^{2}}{2 !} \\
& +\frac{a(a+1)(a+2)}{b(b+1)(b+2)} \frac{z^{3}}{3 !}+\cdots, \\
\sigma & =\frac{\left(\alpha_{1}-\alpha_{2}\right) F_{0}^{2}}{2 k T},
\end{aligned}
$$

and $\alpha_{1}$ and $\alpha_{2}$ are the components of the electric polarizability parallel and perpendicular to the axis of symmetry of the molecule. We remark that all the confluent hypergeometric functions appearing in Eq. (4.14) may be expressed in terms of the more familiar error function of imaginary argument erf $i(x)$. In particular ([18], pp. 580 and 581),

$$
\begin{gathered}
M\left(\frac{1}{2}, \frac{3}{2}, z\right)=\frac{1}{2}\left(\frac{\pi}{z}\right)^{1 / 2} \text { erf } i(\sqrt{z}), \\
M\left(\frac{3}{2}, \frac{7}{2}, z\right)=\frac{15}{8 z^{2}}\left[3 e^{z}-\frac{3+2 z}{2}\left(\frac{\pi}{z}\right)^{1 / 2} \operatorname{erf} i(\sqrt{z})\right] .
\end{gathered}
$$

Equations for the other $M$ functions occurring in Eq. (4.14) may be obtained from Table 7.11.2 of Ref. [18], and the recurrence relations for the confluent hypergeometric function. In our notation the model considered in Refs. [6,7] corresponds to Eq. (4.8) for $n=2$ with

$$
h_{\mathrm{I}}=0, \quad \sigma_{\mathrm{I}}=0, \quad h_{\mathrm{II}}=0, \quad \sigma_{\mathrm{II}}=\sigma,
$$

where according to Eqs. (4.9)-(4.13) and (4.15),

$$
\begin{gathered}
\Phi(z)=\frac{\operatorname{erf} i(\sqrt{\sigma} z)}{2 \operatorname{erf} i(\sqrt{\sigma})}-\frac{z}{2}, \\
\Psi_{2}(z)=\frac{3}{4 \sigma}\left[z e^{\sigma z^{2}}-e^{\sigma} \frac{\operatorname{erf} i(\sqrt{\sigma} z)}{2 \operatorname{erf} i(\sqrt{\sigma})}\right],
\end{gathered}
$$

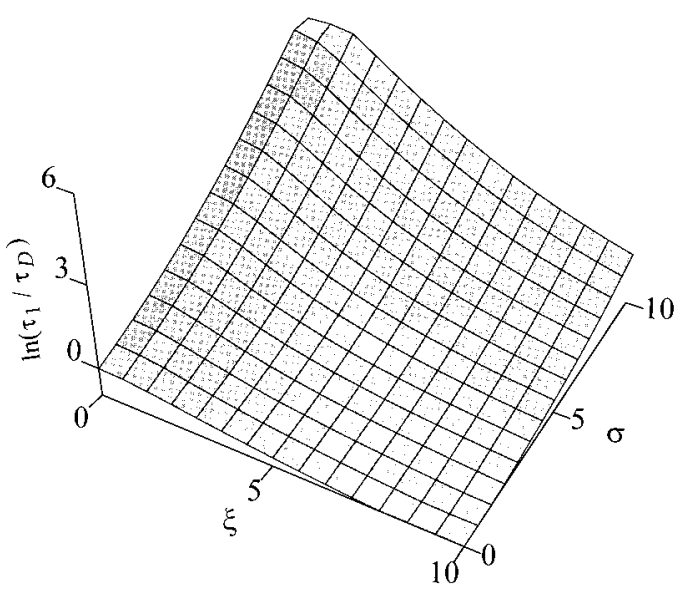

FIG. 1. Nonlinear dielectric relaxation time in the form $\ln \left(\tau_{1} / \tau_{D}\right)$ calculated from Eq. (4.8) as a function of $\sigma$ and $\xi$ for a suddenly reversed dc field: $\xi_{\mathrm{II}}=-\xi_{\mathrm{I}}=\xi$, and $\sigma_{\mathrm{II}}=\sigma_{\mathrm{I}}=\sigma$.

$$
\left\langle P_{2}\right\rangle_{0}=\frac{3}{4 \sigma}\left[\frac{2 e^{\sigma} \sqrt{\sigma}}{\sqrt{\pi} \operatorname{erf} i(\sqrt{\sigma})}-1\right]-\frac{1}{2} .
$$

The result of calculations of the correlation time $\tau_{2}$ from Eq. (4.14) is in complete agreement with that predicted by Eqs. (4.8) and (4.16)-(4.18) (e.g., for $\sigma=5$ we obtained $\tau_{2} / \tau_{D}=0.281269 \ldots$ in both representations). In Ref. [7] we also evaluated the nonlinear dielectric and birefringence rise transients for a more general model of polar and polarizable molecules (the solution was obtained in terms of matrix continued fractions). The model corresponds to

$$
h_{\mathrm{I}}=0, \quad \sigma_{\mathrm{I}}=0, \quad h_{\mathrm{II}}=h, \quad \sigma_{\mathrm{II}}=\sigma .
$$

Here we have also complete agreement between the relaxation times yielded by both solutions.

Equation (4.8) can also be applied to other nonlinear problems of the dynamic birefringence and dielectric relaxation considered by Morita and Watanabe $[4,5]$. In particular, Eq. (4.8) for

$$
h_{\mathrm{I}}=-h_{\mathrm{II}} \quad\left(\text { or } \xi_{\mathrm{I}}=-\xi_{\mathrm{II}}\right), \quad \sigma_{\mathrm{II}}=\sigma_{\mathrm{I}}=\sigma
$$

yields the nonlinear dielectric relaxation time for a transient process where a homogeneous electric field $\mathbf{F}_{0}$ applied to a system of polar and polarizable particles for a time sufficient to allow the system to reach the equilibrium state for $t<0$, is suddenly reversed at $t=0$ (Fig. 1). Furthermore, Eq. (4.8) (for $h_{\mathrm{I}} \neq h_{\mathrm{II}}, \sigma_{\mathrm{II}} \neq \sigma_{\mathrm{I}}$ ) gives the exact solution for the nonlinear birefringence and dielectric relaxation times when a strong homogeneous electric field $\mathbf{F}_{0}$ is suddenly applied to the system where a Maxwell-Boltzmann distribution of the orientations of particles has been established by another homogeneous electric field $\mathbf{F}_{1}$.

Let us now suppose that the amplitude of the strong dc field $\mathbf{F}_{0}$ is suddenly altered at time $t=0$ and that, unlike in Eq. (4.19), the value of the anisotropy parameter $\sigma$ remains unaffected, i.e., we assume

$$
h_{\mathrm{I}} \neq h_{\mathrm{II}}, \quad \sigma_{\mathrm{II}}=\sigma_{\mathrm{I}}=\sigma .
$$




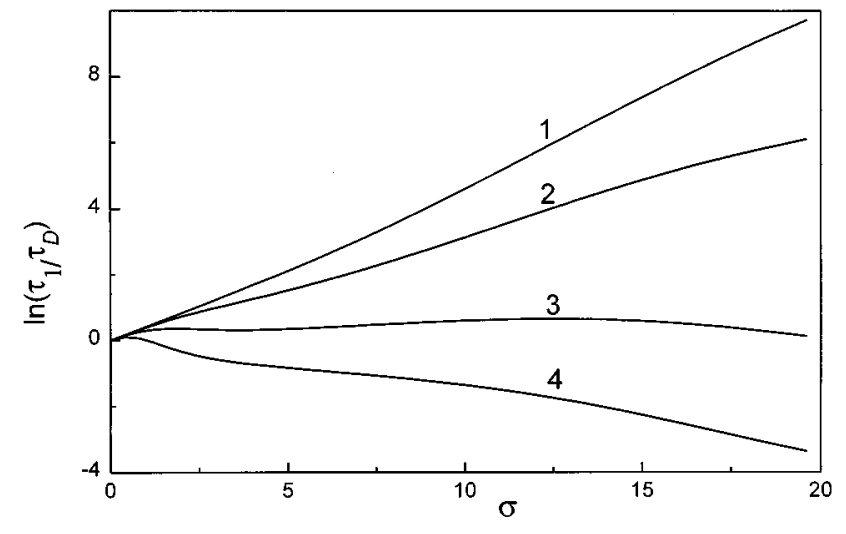

FIG. 2. $\ln \left(\tau_{1} / \tau_{D}\right)$ calculated from Eqs. (4.8) and (4.19) as a function of $\sigma$ for $h_{\mathrm{I}}=0.1 \rightarrow h_{\mathrm{II}}=0.15$ (curve 1), $h_{\mathrm{I}}=0.1 \rightarrow h_{\mathrm{II}}=0.25$ (curve 2), $h_{\mathrm{I}}=0.1 \rightarrow h_{\mathrm{II}}=0.5$, (curve 3), and $h_{\mathrm{I}}=0.1 \rightarrow h_{\mathrm{II}}=1$ (curve 4).

This form of the model is appropriate to the nonlinear dielectric response of nematic liquid crystals and to the nonlinear magnetic response of an assembly of single domain ferromagnetic particles, where $\sigma$ is mainly determined by the crystalline (or shape and magnetic) anisotropy rather than the external field, in contrast to nonlinear dielectric and Kerr effect relaxation of an assembly of permanently polar and polarizable molecules. The linear response of this model has been evaluated elsewhere $[13,16]$ (this corresponds to an infinitesimal change in amplitude of $\mathbf{F}_{0}$ ). The step-on, step-off, and ac linear responses are now entirely determined by the equilibrium dipole autocorrelation function $C_{1}(t)$ $=\langle\cos \vartheta(0) \cos \vartheta(t)\rangle_{0}-\langle\cos \vartheta(0)\rangle_{0}^{2}$ [13], where

$$
\langle(*)\rangle_{0}=\frac{\int_{0}^{\pi}(*) e^{\sigma \cos ^{2} \vartheta+\xi \cos \vartheta} \sin \vartheta d \vartheta}{\int_{0}^{\pi} e^{\sigma \cos ^{2} \vartheta+\xi \cos \vartheta} \sin \vartheta d \vartheta} .
$$

In particular, the relaxation time $\tau_{1}^{\text {lin }}$ of the linear response step-off relaxation function $f_{1}^{\operatorname{lin}}(t) \propto C_{1}(t)$ is [16,21], cf. Eq. (1.6),

$$
\begin{aligned}
\tau_{1}^{\operatorname{lin}}= & \frac{2 \tau_{D}}{\left\langle\cos ^{2} \vartheta\right\rangle_{0}-\langle\cos \vartheta\rangle_{0}^{2}} \int_{-1}^{1} \frac{e^{-\sigma z^{2}-\xi z}}{1-z^{2}} \\
& \times\left[\int_{-1}^{z}\left(z^{\prime}-\langle\cos \vartheta\rangle_{0}\right) e^{\sigma z^{\prime 2}+\xi z^{\prime}} d z^{\prime}\right]^{2} d z
\end{aligned}
$$

where $\langle\cos \vartheta(0)\rangle_{0}=\left\langle P_{1}\right\rangle_{0}$ and $\left\langle\cos ^{2} \vartheta(0)\right\rangle_{0}=\left(2\left\langle P_{2}\right\rangle_{0}+1\right) / 3$ may be readily extracted from Eqs. (4.12) and (4.13), respectively. Equation (4.20) may be obtained from Eq. (4.8) by writing $\xi_{\mathrm{I}}-\varepsilon=\xi_{\mathrm{II}}$, and proceeding to the limit $\varepsilon \rightarrow 0$. Alternative methods of derivation of Eq. (4.20) are given, for example, in Refs. [19-21].

The results of our calculations of the nonlinear step response relaxation time $\tau_{1}$ and comparison with those of the linear response are given in Figs. 2-4: the relaxation time of the rise transient as a function of $\sigma$ for various values of the

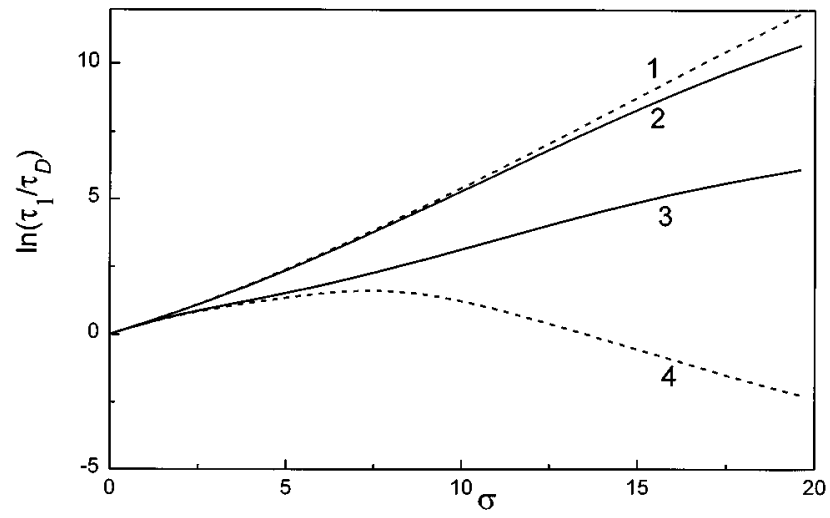

FIG. 3. $\ln \left(\tau_{1} / \tau_{D}\right)$ calculated from Eqs. (4.8), (4.19), and (4.20) as a function of $\sigma$ for linear response $h_{\mathrm{II}}=h_{\mathrm{I}}-\varepsilon=0.1(\varepsilon \rightarrow 0)$ (curve 1 ), nonlinear decay transient $h_{\mathrm{I}}=0.25 \rightarrow h_{\mathrm{II}}=0.1$ (curve 2), nonlinear rise transient $h_{\mathrm{I}}=0.1 \rightarrow h_{\mathrm{II}}=0.25$ (curve 3), and linear response $h_{\mathrm{II}}=h_{\mathrm{I}}-\varepsilon=0.25(\varepsilon \rightarrow 0)$ (curve 4$)$.

bias parameter $h$ is shown in Fig. 2, and the linear and nonlinear relaxation times for the rise and reverse decay transients are shown in Figs. 3 and 4. It is apparent from Figs. 2-4 that the relaxation process obeys an activation (Arrhenius) law behavior (i.e., an exponential increase of $\tau_{1}$ with increasing barrier height $\sigma$ ) in a restricted range of the parameters $\xi$ and $\sigma$ only. This may be explained as follows. The relaxation dynamics in the potential given by Eq. (4.1) (which has in general two potential wells) is determined by two relaxation processes. One relaxation (activation) process governs the crossing of the potential barrier between two positions of equilibrium by a current of particles. Another process describes relaxation inside the wells. Potential (4.1) becomes more and more asymmetrical with increasing $\xi$, and the activation process is suppressed due to the depletion of the upper well [16]. This depletion is achieved at values of the constant electric field $\mathbf{F}_{0}$ which are considerably smaller than the value of a critical field at which the double well

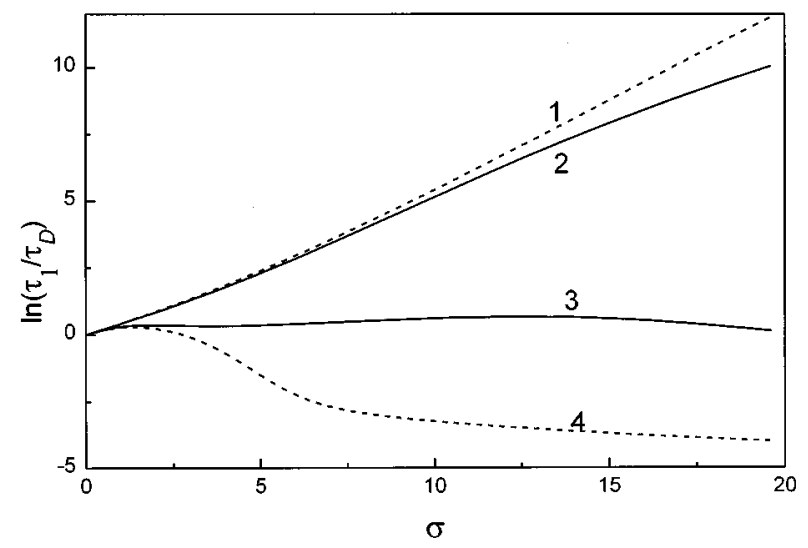

FIG. 4. $\ln \left(\tau_{1} / \tau_{D}\right)$ calculated from Eqs. (4.8), (4.19), and (4.20) as a function of $\sigma$ for a further selection of values of $h$ : linear response $h_{\mathrm{II}}=h_{\mathrm{I}}-\varepsilon=0.1(\varepsilon \rightarrow 0)$ (curve 1$)$, nonlinear decay transient $h_{\mathrm{I}}=0.5 \rightarrow h_{\mathrm{II}}=0.1 \quad$ (curve 2), nonlinear rise transient $h_{\mathrm{I}}=0.1 \rightarrow h_{\mathrm{II}}=0.5$ (curve 3), and linear response $h_{\mathrm{II}}=h_{\mathrm{I}}-\varepsilon=0.5$ $(\varepsilon \rightarrow 0)$ (curve 4$)$. 
structure of the potential disappears (this critical field is given by $\xi / 2 \sigma=1)$. In the case of the strong bias field $(\xi \rightarrow \infty)$, when the potential (4.1) transforms to the single well potential $\xi \cos \vartheta$, we observe intrawell relaxation modes only. Similar results have been obtained for the linear response in magnetic relaxation of single domain ferromagnetic particles with high anisotropy barriers in the presence of a strong constant magnetic field following an infinitesimal change in that field $[13,16,21]$. It is also apparent from Figs. 3 and 4 that nonlinear relaxation times for the step-on $\left(h_{\mathrm{I}} \rightarrow h_{\mathrm{II}}\right)$ and the reverse step-off $\left(h_{\mathrm{II}} \rightarrow h_{\mathrm{I}}\right)$ responses may differ considerably in marked contrast to the linear response step-on and step-off solutions where according to Eq. (1.3) the relaxation behavior is characterized by the same relaxation time. In other words, in linear response the rise and decay transients are mirror images of each other. One can also see in Figs. 3 and 4 that the nonlinear response relaxation times for the step-on
$\left(h_{\mathrm{I}} \rightarrow h_{\mathrm{II}}\right)$ and for the reverse step-off $\left(h_{\mathrm{II}} \rightarrow h_{\mathrm{I}}\right)$ responses are always less than the linear response relaxation time for an infinitesimal change in $h_{\mathrm{I}}$, and are always higher than those for an infinitesimal change in $h_{\mathrm{II}}$ (for $h_{\mathrm{I}}<h_{\mathrm{II}}$ ). This is due to the greater contribution of intrawell relaxation modes to the nonlinear relaxation time than to the linear one.

\section{ACKNOWLEDGMENTS}

One of us (Yu. P. K.) thanks the French Ministry of High Education and Research for a financial assistance (high level Grant PECO-CEI). W.T.C. acknowledges the financial support of the Forbairt Research Collaboration Fund and the French Foreign Office. The partial support of this work by the Russian Foundation for Basic Research (Grant 96-0216762-a) is also gratefully acknowledged.
[1] R. Kubo, J. Phys. Soc. Jpn. 12, 570 (1957).

[2] H. Risken, The Fokker-Planck Equation, 2nd ed. (Springer, Berlin, 1989).

[3] A. Morita, Phys. Rev. A 34, 1499 (1986).

[4] A. Morita and H. Watanabe, Phys. Rev. A 35, 2690 (1987).

[5] H. Watanabe and A. Morita, Advances in Chemical Physics, edited by I. Prigogine and S. A. Rice (Wiley, New York, 1984), Vol. 56, pp. 255-409.

[6] J.-L. Déjardin, P. Blaise, and W. T. Coffey, Phys. Rev. E 54, 852 (1996).

[7] W. T. Coffey, J. L. Déjardin, Yu. P. Kalmykov, and S. V. Titov, Phys. Rev. E 54, 6462 (1996).

[8] J.-L. Déjardin, Dynamic Kerr Effect (World Scientific, Singapore, 1995).

[9] W. T. Coffey, Yu. P. Kalmykov, and J. T. Waldron, The Langevin Equation (World Scientific, Singapore, 1996).

[10] A. J. Martin, G. Meier, and A. Saupe, Symp. Faraday Soc. 5, 119 (1971).

[11] W. T. Coffey, D. S. F. Crothers, Yu. P. Kalmykov, and J. T.
Waldron, Physica A 213, 551 (1994).

[12] W. F. Brown, Jr., Phys. Rev. 130, 1677 (1963).

[13] W. T. Coffey, D. S. F. Crothers, Yu. P. Kalmykov, and J. T. Waldron, Phys. Rev. B 51, 15947 (1995).

[14] Yu. L. Raikher and M. I. Shliomis, Advances in Chemical Physics, edited by W. T. Coffey (Wiley, New York, 1994), Vol. 87, p. 595.

[15] W. T. Coffey, D. S. F. Crothers, Yu. P. Kalmykov, E. S. Massawe, and J. T. Waldron, Phys. Rev. E 49, 1869 (1994).

[16] D. A. Garanin, Phys. Rev. E 54, 3250 (1996).

[17] Handbook of Mathematical Functions, edited by M. Abramowitz and I. Stegun (Dover, New York, 1965).

[18] A. P. Prudnikov, Yu. A. Brychkov, and O. I. Marichev, Integrals and Series. Additional Chapters (Science, Moscow, 1986).

[19] A. Szabo, J. Chem. Phys. 72, 4620 (1980).

[20] G. Moro and P. L. Nordio, Mol. Phys. 56, 255 (1985).

[21] W. T. Coffey and D. S. F. Crothers, Phys. Rev. E 54, 4768 (1996). 